\title{
Die Renaissance der Luftbrücke
}

A. Gabel Analyse der Evakuierungsoperation von Phuket, Thailand

\author{
The Renaissance of the Air Bridge \\ Analysis of the Evacuation Operation in Phuket, Thailand
}

\section{Zusammenfassung}

Das Seebeben im indischen Ozean am 26.12.2004 hat eine der größten zivilen Katastrophen seit dem Ende des 2. Weltkrieges ausgelöst. Bis heute ist die genaue Anzahl Verletzter, Vermisster und Toter unbekannt. Aus notfallmedizinischer Sicht zog die Flutwelle den umfangreichsten und logistisch schwierigsten Einsatz nach sich, den die beteiligten Hilfsorganisationen der Bundesrepublik Deutschland je zu bewältigen hatten. Dabei rückten die Besonderheiten der Schadenslage und logistischen Rahmenbedingungen flugmedizinischer Aspekte stark in den Vordergrund. Für das Management eines Großschadensereignisses derartigen internationalen Ausmaßes erwies sich die Einbindung der drei Säulen deutscher Flugrettung - Deutsche Lufthansa AG, Deutsche Bundeswehr und der Ambulanzfluggesellschaften - als unabdingbar: Rund 2500 obdachlos gewordene oder verletzte Touristen mussten mittels arztbesetzter Sonderflüge unverzüglich evakuiert werden. Von den 300 Schwerverletzten wurden 200 durch die Deutsche Lufthansa AG, rund 100 durch die Bundeswehr betreut und binnen 6 Tagen erfolgreich repatriiert. Damit hatte Deutschland als erste Nation ihr selbst gestecktes Ziel erreicht. Ein derartiges logistisches Meisterstück war insbesondere der schrankenlosen und organisationsübergreifenden $\mathrm{Zu}$ sammenarbeit aller beteiligten Hilfskräfte zu verdanken. Die Autoren möchten die Leistung aller Beteiligten würdigen und ihre Erfahrungen für dringend erforderliche Verbesserungen in der Organisation des deutschen und internationalen Katastrophenschutzes zur Verfügung stellen.

Schliuisselwörter

Phuket · Evakuierung · Erdbeben · Katastrophe · Luftrettung

\section{Abstract}

The earthquake in the Indian Ocean on 2004-12-26 was followed by one of the greatest civilian catastrophes since the second world war. Up to now the exact number of injured, missed or dead humans is uncertain. From a disaster-medicine point of view the flood-wave led to the logistically most difficult and extensive mission, German forces and health-care-organisations ever had to cope with. Due to the special circumstances and logistic requirements of the disaster, aviation medicine played a crucial role in its solution. The management of a catastrophe with such an international extent would not have been possible without extensive involvement of the three columns of German air-rescue - Lufthansa German Airlines, German Airforces and ambulance-jet-companies. About 2500 homeless or injured tourists had to be immediately evacuated by physician-accompanied special flights. From a total of 300 seriously injured persons 200 were treated and repatriated within 6 days by Lufthansa, about 100 with the German Airforces. By that Germany was the first nation to reach her self-claimed aim. Such a logistic masterpiece must be attributed and honoured to the limitless collaboration of all involved organisations and volunteers. The authors explicitly want to appreciate performance and success of all attributors and offer their current experience for improvements regarding the organisation of German and international disaster management, that have shown to be required urgently.

\section{Key words}

Phuket - evacuation operation - earthquake · disaster - air rescue 
Einleitung

In Deutschland brannten die Weihnachtskerzen und die Menschen genossen die häusliche Geborgenheit im Kreis ihrer Familien, als im fernen Ferienparadies Thailand eine mörderische Bedrohung auf die Küsten zurollte. Ihrer Neugierde folgend wurden ahnungslose Kinder und Erwachsene vom zurückweichenden Meereswasser geradewegs in eine Todesfalle gelockt. Doch auch vorsichtige Badegäste, welche noch rechtzeitig die Flut vorausahnten, hatten kaum eine Chance zur Flucht: In ebenem Gelände erreichte die Flutwelle eine derartige Wucht, dass sie selbst nach einer Auslaufstrecke von $500 \mathrm{~m}$ bewaldetem Gelände Straßen überquerte, Häuserwände einriss und Lastwagen in Binnenseen versenkte.

Die meisten Menschen waren sofort tot. Der Strand war nach dem Abfließen der Wassermassen von Leichen übersät. Wer von den Überlebenden noch die Kraft hatte, in den Fluten nach seinen Angehörigen zu tasten, bekam häufig nur noch ein totes Kind zu greifen. Fast ausschließlich Erwachsene hatten überleben können. Eine Reihe von ihnen berichtete von einem schrecklichen Todeskampf. Das Ertrinken haben sie über Minuten hin bei vollem Bewusstsein erlebt: Die Lungen voller Meerwasser und Sand versuchten sie mit aller Kraft mehrere Male „nach oben“ zu kommen, bis schließlich die Kraft ausging und ein Gefühl der Resignation einsetzte: „ich kann nicht mehr, dann sterbe ich halt" war der Gedanke, mit dem viele zuletzt ihr Leben losließen. Nur wenige durften danach weiterleben. Das seelische Trauma, welche sie aus dieser Erfahrung tiefster und hartnäckiger Lebensbedrohung mit sich herumtragen müssen, ist unvorstellbar.

Die Schadenslage war medizinisch gekennzeichnet durch ein Alles-oder-Nichts-Gesetz. Die Wucht der Flutwelle imponierte bei einem Gewicht von einer Tonne pro Kubikmeter Wasser und einer Laufgeschwindigkeit von bis zu $800 \mathrm{~km} / \mathrm{h}$ so stark, dass sie nur überlebt werden konnte, wenn keine direkte Exposition gegenüber der Wellenfront bestand. Dementsprechend fanden sich überwiegend leicht verletzte Überlebende und nur wenig Schwerverletzte. Die Mehrzahl der Leichen war durch eine großflächige Gewalteinwirkung derart entstellt, dass selbst eine Unterscheidung zwischen Europäern und Asiaten nicht mehr gelang. Selbst bei bester Kenntnis persönlicher Gesichtszüge konnte keine Identifikation mehr erfolgen. Flächige verfärbte Hautblutungen und Gewebsquellung ließen den Rückschluss auf eine diffuse Krafteinwirkung und Ertrinken als Todesursache zu. Leblose Körper fanden sich zu Tausenden über einen Küstenstreifen von ca. $15 \mathrm{~km}$ verteilt. Im Ferienresort Khao Lak waren 13000 Hotelbetten überwiegend mit Deutschen und Schweden voll belegt. Kaum ein Stein ist dort auf dem anderen geblieben. Die meisten der Gäste hatten sich zum Zeitpunkt der Katastrophe am Strand aufgehalten.

Die Überlebenden wiesen in der Mehrzahl Extremitätenverletzungen auf. Es fanden sich tiefe Fleischwunden mit großen Substanzdefekten. Am Rumpf dominierten Schürfwunden, Hämatome und Rippenfrakturen. Die Betroffenen waren von den Wassermassen über weite Strecken mitgerissen und gegen Gebäudeteile, Vegetation oder Mobiliar geschleudert worden. Dementsprechend überwogen die beschriebenen Schleif- und Anpralltraumata. Von den inneren Organen zeigte sich die Lunge am häufigsten betroffen. Salzwasser- und Sandaspiration führten schnell zu schweren Pneumonien und ARDS mit respiratorischer Insuffizienz. Als Komplikation traten in dem tropischen Klima bei fast allen Patienten binnen kürzester Zeit schwerste anaerobe Wundinfektionen und therapierefraktäre Phlegmonen auf. Trotz adäquater 3-fach-Antibiose drohte in einer Vielzahl ein infektionsbedingtes Compartmentsyndrom und für die meisten Verletzten tickte die „septische Zeitbombe“.

Die Primärrettungsphase ist vollständig und in perfekter Weise durch die thailändischen Militärs und Hilfskräfte abgewickelt worden. Europäische Hilfe traf viel zu spät ein, um einen relevanten Beitrag leisten zu können. Innerhalb des 1. und 2. Tages sind alle Überlebenden von Rettungsmannschaften und Hubschraubern aufgesammelt oder aus dem Meer gefischt worden. Sie wurden in Krankenhäuser in einem Umkreis bis zu 1000 km gebracht und den Umständen entsprechend vorbildlich akutmedizinisch versorgt. Thailändische Krankenhäuser haben bevorzugt ausländische Opfer vor ihren eigenen Landsleuten behandelt. Trotz maximaler Überlastung fanden wir Organisation, Diagnostik, Therapie und menschliche Betreuung optimal vor. Die Ausstattung der Kliniken und die Kompetenz des Fachpersonals überflügelte häufig deutsche Standards, welche mittlerweile unter der Sparpolitik des letzten Jahrzehntes den internationalen Vergleich scheuen müssen. Hervorzuheben ist die unglaubliche Freundlichkeit und Hilfsbereitschaft der einheimischen Ärzte, Schwestern und Laienhelfer. Trotz vorbildlicher Versorgung tat die Evakuierung der zahlreichen Patienten Not, da die Überlastung der örtlichen Gesundheitsstrukturen eine zeitaufwändige Weiterbehandlung, wie regelmäßige Wundnachsorge, Debridement und individuelle Intensivtherapie, bei der hohen Anzahl ausländischer Patienten kaum ermöglichte.

Die Sekundärrettung aus Deutschland begann mit dem Eintreffen der ersten Ärzte des Medizinischen Dienstes der Lufthansa und einem 15-köpfigen Bergetrupp des THW auf einem Sonderflug der Condor am Abend des 28.12.2004 sowie Ärzten des ADAC, zwei Tage nach der Katastrophe. Weitere Ärzte und personelle Unterstützung des Auswärtigen Amtes gelangten am Folgetag, dem 29.12. nach Phuket. Den ersten Helfern standen so gut wie keine Informationen zur Verfügung. Der mutmaßlichen Schadenslage entsprechend beschlossen die Notfallmediziner, die Sekundärrettung in zwei Phasen mit unterschiedlicher Zielrichtung abzuarbeiten. Beide Phasen waren wesentlich von flugmedizinischen und logistischen Erfordernissen geprägt. Die medizinische Behandlung stand demgegenüber nach abgeschlossener Primärversorgung weitgehend im Hintergrund.

Phase 1 der Sekundärrettung hatte einen möglichst hohen Umsatz an unverletzten oder leicht verletzten Passagieren zum Ziel. Mehrere tausend Überlebende waren schlagartig obdachlos geworden und seelisch massiv traumatisiert. Ernährung und hygienische Versorgung wurden zu einem Problem, welches innerhalb kürzester Zeit nach einer Lösung verlangte. Andernfalls drohte eine Seuchenlage und chaotische Verhältnisse. Der Massenanfall an Patienten stand im krassen Missverhältnis zur Anzahl des medizinischen Fachpersonals und Fehlen jeglicher medizinischer Infrastruktur. Das Eintreffen weiterer Hilfskräfte und brauchbaren Equipments verlief - gemessen an den Sacherfordernissen schleppend und zog sich über die nächsten Tage hinweg. 
Erschwerend trat hinzu, dass keinerlei Einschätzung zur Zahl der Überlebenden möglich war. Die Verbindungen zu den Ferienresorts waren unterbrochen. Von den 13 bekannten Reiseleitern galten vier als tot oder vermisst. Die Reiseveranstalter konnten keine Angaben zum bevorstehenden Beförderungsbedarf machen. Die Einschätzungen der überlasteten und mit einem medizinischen Katastrophenmanagement fachlich überforderten Behörden liefen aus notärztlicher Sicht zumeist den tatsächlichen Ereignissen weit hinterher und mussten als unrealistisch eingestuft werden. So war von offizieller Seite noch tagelang von „vier deutschen Toten" die Rede, obwohl das Desaster in Khao Lak, $80 \mathrm{~km}$ im Norden Phukets, für jedermann ab der ersten Stunde ersichtlich wurde.

Der Krisenstab der Condor sowie des medizinischen Dienstes der Lufthansa in Frankfurt unter der Leitung von Herrn Professor Stüben beschlossen in Anbetracht der augenscheinlichen Großschadenslage die sofortige Entsendung von acht Sonderflügen mit Hilfsgütern, Ärzten und Schwestern des Medizinischen Dienstes auf Kosten des Konzerns. Die freiwillige Vorleistung der Fluggesellschaften bedurfte keiner bürokratischen Genehmigung staatlicher Stellen. Sie stützte sich auf die Expertise eines krisenmedizinisch sehr erfahrenen und fachärztlich breit gefächerten Ärztestabes, welcher bereits maßgeblich an der Bewältigung von Großschadensereignissen (z.B. Flugzeugabsturz in Warschau, Flugzeugentführungen) und internationaler Krisen (Pestepidemie in Indien, SARS-Ausbruch in Asien) beteiligt war. Diese günstigen organisatorischen Rahmenbedingungen führten dazu, dass das erste Großraumflugzeug der Condor nach zwei Tagen, die erste Medevac-Maschine der Bundeswehr vier Tage nach der Katastrophe in Phuket eintraf. Unter Berücksichtigung der sachlichen Erfordernisse einer medizinischen Akutversorgung erscheint uns eine derartige zeitliche Verzögerung nicht akzeptabel und organisatorisch verbesserungswürdig.

Glücklicherweise gerieten Passagieraufkommen am Flughafen und Zahl der eintreffenden Großraumflugzeuge nicht in ein Missverhältnis, sodass eine geregelte Passagierabfertigung möglich wurde. Problematisch war dabei die Erfassung der Passagiere und Erledigung der Ausreiseformalitäten, da viele ihre Reisedokumente und Pässe verloren hatten. Damit wurde ein Abgleich zwischen Einreise- und Ausreisedaten als Quellinformation für die Anzahl Vermisster unbrauchbar. Die thailändischen Behörden und die deutsche Botschaft regelten Identitätsnachweis und Ausreiseformalitäten den Erfordernissen entsprechend sehr großzügig, sodass der Passagierfluss nicht behindert wurde.

Die ärztliche Versorgung der Passagiere konnte sich in dieser Phase auf die flugmedizinische Beurteilung der Flugreisetauglichkeit beschränken. Dabei galt es insbesondere Erkrankungen zu beachten, welche sich unter Druckabfall oder milder Hypoxie verschlimmern konnten: so bergen beispielsweise ein unerkannter Pneumothorax oder eine beginnende respiratorische Insuffizienz eine große Gefahr für Akutkomplikationen während des Fluges. Die Möglichkeiten zur medizinischen Intervention sind an Bord naturgemäß begrenzt und je nach Flugposition kann es Stunden dauern, bis eine Zwischenlandung an einem Flughafen mit adäquater klinischer Versorgungsmöglichkeit möglich ist. Eine derartige Zwischenlandung erfordert zumeist das zuvorige „Dumpen“ von bis zu 100 Tonnen Treibstoff in die Atmosphäre, um das maximal zulässige Landegewicht zu erreichen. Eine derartige Entscheidung will nicht zuletzt aus ökologischen Gründen gut begründet sein.

Mit dem annähernd komplikationsfreien Heimtransport von ca. 2500 leicht oder unverletzten Passagieren durch die arztbesetzten Flugzeuge konnte die Phase 1 innerhalb von drei Tagen erfolgreich abgeschlossen werden. Nur Einzelne mussten vom boarding abgehalten und zunächst klinisch versorgt werden. Lediglich ein Patient wurde wegen Rhinoliquorrhö und progredienter klinischer Verschlechterung bei einer Zwischenlandung von Bord gebracht. Etwa 160 freiwillige Mitarbeiter der SAT (Special Assistance Teams der Lufthansa) versorgten die ankommenden Passagiere in Frankfurt und München mit Lebensmitteln oder Kleidung und betreuten sie mittels Krisenintervention. Das Auffanglager am Flughafen Frankfurt wurde maßgeblich durch Ärzte und Fachpersonal der FRAPORT AG unter der Leitung von Herrn Dr. Gaber betreut. Die logistischen Leistungen waren spiegelbildlich zu den Aufgaben des entsendenden Lagers in Phuket.

Phase 2 der Sekundärrettung konnte in Angriff genommen werden, als am 29.12.2004 medizinische Verstärkung aus Deutschland eintraf. Eine Reihe von niedergelassenen Ärzten, Kollegen aus dem Krankenhaus Schwabing sowie Ärzte und Rettungsassistenten des Bayrischen Roten Kreuzes bildeten mit Unterstützung der Deutschen Botschaft eine schlagkräftige Einsatzgruppe. Am Flughafen hatte die thailändische Regierung für alle betroffenen Nationen eine Halle zur Verfügung gestellt, welche als Verbandplatz und Sammellager genutzt werden konnte. Alle Beteiligten waren notfallmedizinisch überaus erfahren. Herr Kollege Vorderwülbecke hatte zahlreiche Auslandseinsätze mit der Bundeswehr geleistet (Afghanistan, Kosovo) und stand als Bindeglied zum Medevac-Team zur Verfügung. Herr Braun, ebenso ehemaliger Medevac-Arzt, verfügte als Ärztlicher Leiter der DRF über profunde logistische und flugmedizinische Kenntnisse sowie eine große Erfahrung im Umgang mit Militär/Bundeswehr, ausländischen Behörden und Strukturen. Herr Gabel hatte die Evakuierungsoperation von Anfang an vor Ort betreut und verfügte als ärztlicher Vertreter der Lufthansa und Flugmediziner über einen unmittelbaren Zugriff auf nachrückende Großraumflugzeuge und die lokale Infrastruktur des Flughafens.

Wie es sich herausstellte, waren es insbesondere die persönlichen Kontakte und Einflussmöglichkeiten der drei Genannten auf die Säulen der Luftrettung, welche einen reibungslosen Ablauf der Evakuierungsoperation ermöglichten. Oftmals erwies sich der offizielle Weg über die Behörden und zahlreichen Instanzen als viel zu Zeit raubend. Ein kurzes Telefonat mit einem befreundeten Kollegen regelte das meiste in Minuten, was über den bürokratischen Weg sonst Stunden bis Tage gedauert hätte. Es ist in allen Lebenslagen ein wichtiges Prinzip, dass ein gegenseitiges persönliches Kennen ein tragfähiges Vertrauen und eine gute Arbeitsgrundlage schafft. Dieser Grundsatz hat sich auch im Management einer derartigen Katastrophe bewährt.

Dementsprechend übernahmen die drei Verbindungsleute die ärztliche Koordinierungs- und Leitungsfunktion am Flughafen. Die ärztliche Gesamtleitung oblag der Regionalärztin des Auswärtigen Amtes. Ihr Diplomatenstatus erforderte ihre überwiegende Präsenz in den zahlreichen Krankenhäusern, sodass sie 
für die Stabsarbeit am Flughafen kaum zur Verfügung stehen konnte. In Anbetracht der hervorragenden Zusammensetzung des Ärzteteams mit durchweg sehr routinierten und selbstständigen Fachärzten war eine fachliche Leitung vollkommen entbehrlich. Übereinstimmend fand die gesamte Ärztegruppe, noch nie so gut, motiviert und freundschaftlich mit Kollegen zusammengearbeitet zu haben, ohne dass irgendeine Art von Hierarchie erforderlich gewesen wäre.

Nicht bewährt hat sich aus unserer Sicht die fast ausschließliche Besetzung der örtlichen Einsatzleitung durch Mitarbeiter des KIT nach Beauftragung durch das Auswärtige Amt. Obgleich eine Reihe der KIT-Kräfte eine Doppeltqualifikation als Rettungssanitäter, -assistent oder OrgLeiter mitbrachte, war die medizinische und ärztliche Repräsentanz im Einsatzstab damit unzureichend. Als die Kommunikationsmöglichkeiten des Stabes unter der Flut der zu verarbeitenden Informationen einen kritischen Punkt erreichte, konnten medizinische Erfordernisse und Eingaben häufig nicht mehr adäquat an die übergeordneten Entscheidungsträger weitervermittelt werden. Umgekehrt sahen sich die Ärzte einem chronischen Informationsrückstand ausgesetzt. Hierdurch war aus unserer Sicht der Erfolg einer Reihe von Einzeloperationen gefährdet. In der Konsequenz koppelte sich die Arztgruppe zeitweise von der Einsatzleitung ab und regelte die Geschehnisse auf informellem Weg selbst. Dies stellte sicher keine optimale Strukturierung des Miteinander dar, blieb aber die einzige Möglichkeit, dank der beispielhaften Unterstützung einzelner Botschaftsmitarbeiter und des sehr kompetenten und kooperativen Militärattachés, das hervorragende Gesamtergebnis der Evakuierungsmaßnahmen zu bewahren. In einer zukünftigen Schadenslage wäre aus unserer Sicht zu fordern, alle maßgeblichen Fachgruppen paritätisch an der Stabsarbeit vor Ort zu beteiligen und die Schnittstellen möglichst mit Spezialisten verantwortlich zu besetzen. Dessen unbenommen sei den KIT-Kräften für ihre hervorragende und unermüdliche Organisationsarbeit gedankt. Das Ausmaß der abzuarbeitenden Informationsflut und Widrigkeiten hätte in Anbetracht der begrenzten Mittel auch den erfahrensten Krisenstab überfordert.

Die wesentliche Leistung, nämlich Sichtung und Abtransport der Schwerverletzten aus den zahlreichen Krankenhäusern zum Flughafen, erbrachten die einzelnen Scout-Teams, zumeist besetzt durch je einen Arzt und Rettungsassistenten. Alleine um Phuket Town galt es, ca. 30 Kliniken zu durchforsten. Anfänglich standen kaum Fahrzeuge zur Verfügung, doch in unermüdlicher Organisations- und Feinarbeit wurden alle Krankenhäuser besucht und die Patienten unbeschadet zum Sammellager verlegt. Dort musste ihre Ankunft eng mit dem Eintreffen der Flugzeuge koordiniert werden, um unnötige Wartezeiten in der behelfsmäßig eingerichteten Halle zu vermeiden (Abb.1). Eine medizinische Versorgung war dort kaum zu realisieren, und auch einfache hygienische Bedürfnisse stießen auf organisatorische Schwierigkeiten. Von flugbetrieblicher Seite war zu beachten, dass ein Flugzeug nur wenige Stunden zum Beladen zur Verfügung stand, bevor es unaufschiebbar den Rückflug antreten musste, da andernfalls die maximal zulässige Arbeitszeit der Piloten überschritten wurde. Der Antritt eines Fluges wäre dann nicht mehr statthaft gewesen und die Patienten hätten wieder entladen werden müssen.

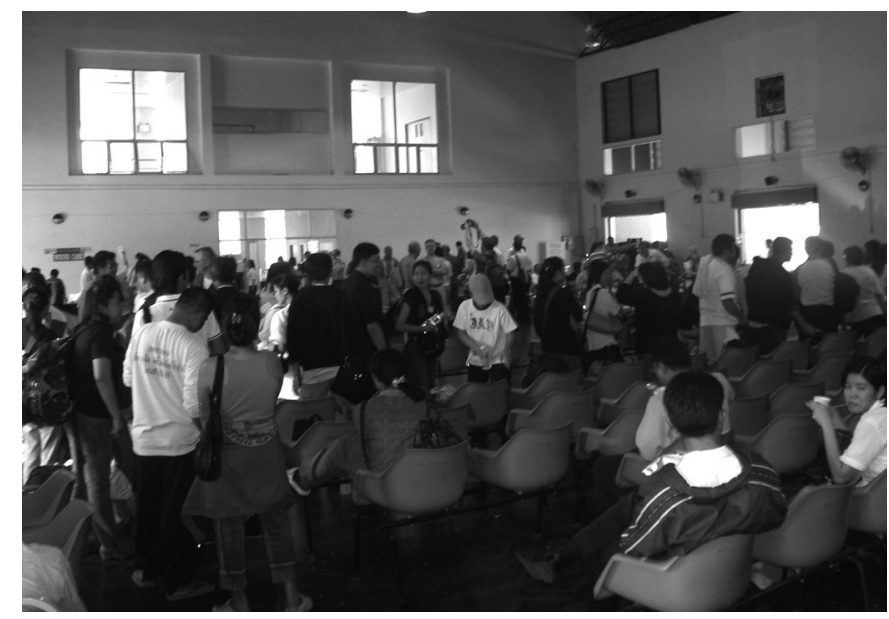

Abb. 1 Presserummel in einem Auffanglager, das aus allen Nähten platzt. Eine diskrete medizinische Individualversorgung ist kaum mehr möglich. Nur unter Mühe gelingt es, eine überschaubare Raumordnung des Verbandsplatzes aufrechtzuerhalten.

Auch die 2. Phase der Sekundärrettung verlief äußerst erfolgreich. Am 6. Tag nach der Katastrophe waren alle Krankenhäuser Phukets und Südthailands von ausreisewilligen und verlegungsfähigen deutschen Patienten frei. Von den ca. 300 hospitalisierten Schwerverletzten wurden 200 durch Spezialflugzeuge der Lufthansa und etwa 100 durch das Ambulanzflugzeug der Bundeswehr ausgeflogen. Daneben wurden einige Individualtransporte mittels Lear-Jets der Ambulanzfluggesellschaften durchgeführt. Die Linienflugzeuge der Lufthansa und Condor waren mittels Stretchern (Flugkrankentragen) und Notfallequipments ausgerüstet worden (Abb. 2). Eine Boing 767 bot innerhalb weniger Stunden Aufrüstzeit Platz für 20 Stretcher und weitere 30 Liegendtransporte auf Business-Class-Sitzen. Teams aus 5 Ärzten und 10 Rettungsassistenten hielten auf einem derartig ausgestatteten Linienflugzeug die Kapazität für den Transport von bis zu zehn Beatmungspatienten vor. Für die hoch qualifizierte personelle und materielle Hilfe sei insbesondere einer Gruppe Frankfurter Notärzte sowie den Mitarbeitern der Berufsfeuerwehr Frankfurt gedankt.

Hervorzuheben ist die spontane und solidarische internationale Zusammenarbeit. Als bemerkenswertes Beispiel möchten wir die Evakuierung von 20 Patienten, 5 hiervon schwer verletzt und respiratorisch instabil, aus dem $200 \mathrm{~km}$ entfernten Hat Yai am 1.1.2005 nennen: Nach diplomatischer Anbahnung durch Herrn Oberst Ibrom stand ein amerikanischer Hercules C 130 Militärtransporter zur Verfügung, mit welchem der für den A 310 Medevac nicht anfliegbare Flughafen von Hat Yai erreichbar wurde. Das gegenseitige interessierte Bestaunen der unterschiedlichen Militärmaschinen wurde für Amerikaner wie Deutsche zu einem echten Erlebnis und half, Freundschaften zu knüpfen. Bei der anschließenden Evakuierungsoperation mit drei medizinischen Notfallsituationen bereits während der Startphase lief die Zusammenarbeit der nicht miteinander vertrauten Mannschaften derart Hand in Hand, dass beide sich völlig voneinander beeindruckt zeigten und gemeinsam alle Schwierigkeiten komplikationsfrei meisterten. Die reibungslose Patientenübergabe an die zweite Medevac am 1.1.2005 beendete die Operation, und Südthailand war von verlegungsfähigen deutschen Verletzten frei (Abb. 3 u. 4). 


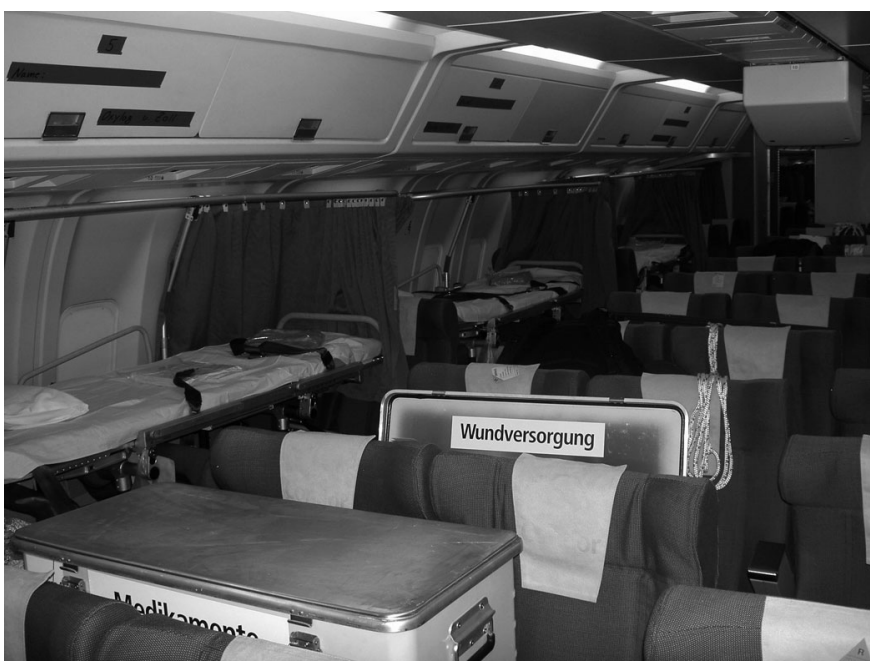

Abb. 2 Eine Boing 767 der Condor oder Lufthansa kann innerhalb von wenigen Stunden mit 20 Stretchern ausgerüstet werden. Daneben stehen 30 Business-Class-Sitze für Liegendtransporte zur Verfügung. Bis zu 10 Beatmungsplätze können betreut werden. Das Flugzeug erreicht ohne Zwischenlandung die meisten fernen Destinationen. Somit bietet ein Linienflugzeug unter Betreuung durch den Medizinischen Dienst der Deutschen Lufthansa AG die derzeit größte Transportkapazität für einen Massenanfall von Verletzten.

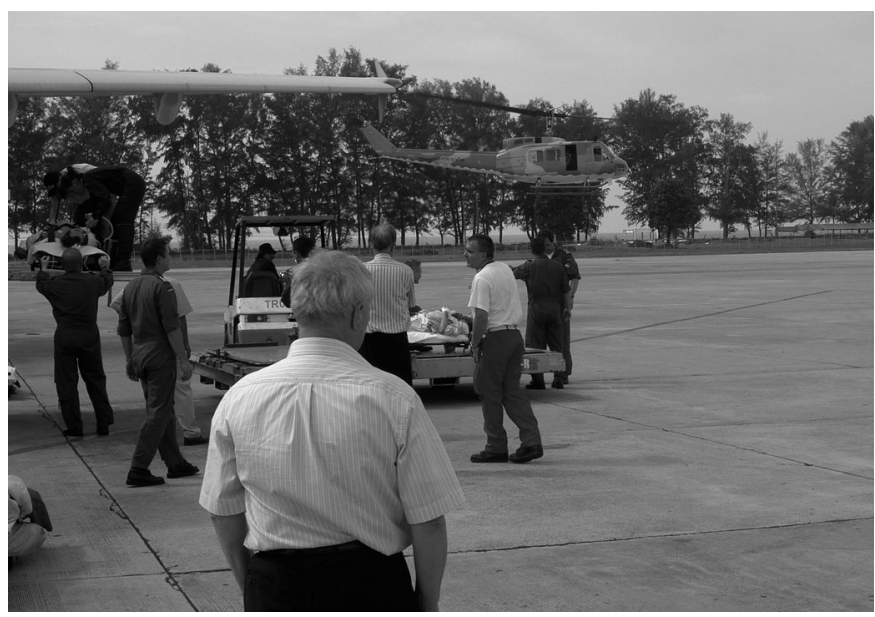

Abb. 3 Die Zeit drängt. Direktes Umladen von Beatmungspatienten aus einer Bell UH 1 D der thailändischen Streitkräfte in den Airbus A 310 Medevac.

Die Analyse der Evakuierungsoperation führt zu dem Ergebnis, dass alle Beteiligten unter höchstem persönlichen Einsatz und schwierigsten äußeren Bedingungen Hervorragendes geleistet und das bestmögliche Ziel erreicht haben. Diese Feststellung treffen zu können ist keine Selbstverständlichkeit und ein Geschenk des Schicksals. Sie gilt gleichermaßen für die Einsatzkräfte vor Ort wie für die vielen Helfer und Angehörigen zu Hause, die Crews der zahlreichen Hilfsflugzeuge sowie Politiker, Militärs und Diplomaten in den Krisenstäben. Alle haben ihr Bestes gegeben. Unser persönlicher Dank gilt vor allem den Kollegen Herrn Dr. med. Platiel und Frau Dr. med. Handreke vom Auswärtigen Amt, welche mit höchstem persönlichen Einsatz und fachlicher Qualifikation erforderliche Entscheidungen top down aus dem Krisenzentrum in Berlin oder vor Ort stets unverzüglich umsetzten. Daneben haben insbesondere Herr Oberst Ibrom, Militärattaché in Asien, sowie Herr Reichert (Deutsche Botschaft, Bang-

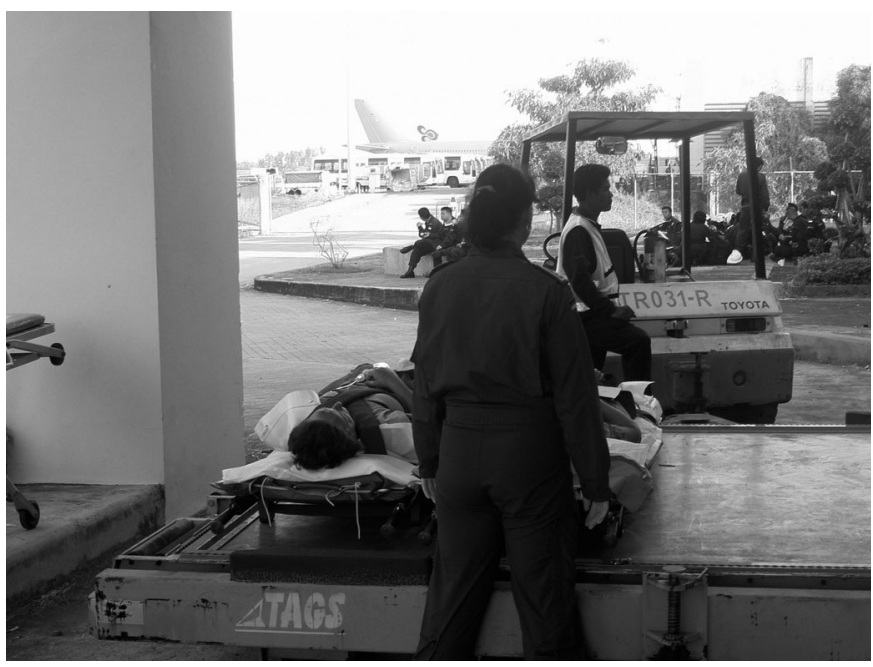

Abb. 4 Heiße Temperaturen und Fehlen von Ambulanzfahrzeugen werden durch Improvisationsgabe und unkonventionelles groundhandling wettgemacht.

kok) und Herr Boling (Stationsleiter Lufthansa, Bangkok) unschätzbare Dienste diplomatischer und organisatorischer Art geleistet.

Dennoch möchten wir einige kritische Anmerkungen treffen, die sich allerdings keinesfalls auf einzelne Personen, sondern auf strukturelle Mängel beziehen. Jeder Mensch kann nur so gut sein, wie die Strukturen, in die er eingebunden ist. Diesbezüglich stellen wir ein dringend korrekturbedürftiges Organisationsdefizit des deutschen und internationalen Katastrophenschutzes fest, welches in einer ähnlichen Schadenslage ebenso zum Verhängnis hätte werden können.

Der erste Kritikpunkt trifft die verspätete Wahrnehmung der Katastrophe in ihrem ganzen Ausmaß und Entwicklung eines adäquaten Problembewusstseins. Bei kaum einer Gelegenheit war das Ausmaß für Fachleute wie Laien bei Ansicht der ersten Fernsehbilder am 27.12.2004 derart evident, wie bei diesem Ereignis. Unsere Notärzte befanden einhellig, dass ihre erste Reaktion am Morgen des 27.12.2004 die unverzügliche Entsendung zweier Medevac-Flugzeuge und eines Luftfrachters mit Lazarettmaterial gewesen wäre. Die Verhältnisse vor Ort haben ihnen Recht gegeben. Aus unserer Erfahrung haben eine Reihe von Politikern und Diplomaten aufgrund ihrer anders gelagerten Aufgabenfelder und Erfahrungshintergründe eine völlig andere Wahrnehmung der Dinge und Handlungsstrategien als katastrophenmedizinisch erfahrene Ärzte. Die Tendenz, Probleme herunterzuspielen, gipfelte im tagelangen Beharren der Meldung, es gebe vier deutsche Tote. Diese Aussage war auf der Sachebene der Leichenidentifikation sicher richtig, ging aber bizarr an der wahren Lage und den Handlungserfordernissen vorbei.

Krisenstäbe traten verspätet zusammen. Katastrophenmedizinisch erforderliche Beschlüsse wurden aus unserer Sicht anfänglich nur zögerlich umgesetzt. Bevor - zu zaghaft - ein Bergetrupp des THW als erste Hilfsreaktion auf den Weg geschickt wurde (Alarmierung ca. $24 \mathrm{~h}$ nach dem Ereignis), wurde zu lange und mit ungenügender Effizienz versucht, sich erst einmal ein Bild der Lage zu verschaffen. Die abwartende diplomatische Haltung 
hatte viel Zeit gekostet. Das Lagebild von Khao Lak wurde dem deutschen Krisenstab erst nach 24 Stunden bekannt. Als die ersten offiziell entsandten Ärzte eintrafen, waren drei Tage vergangen. Diese Ärzte standen anfänglich „barfuß“ in einem fremden Land, ohne katastrophentaugliches Equipment oder Infrastrukturen, wenn sie sich nicht aus eigener Veranlassung mit einigen Notfallrucksäcken zur Individualversorgung ausgestattet hätten. Vom Aufbau eines Lazaretts und Basislagers zur effizienten Versorgung einer Vielzahl von Verletzten waren die Helfer weit entfernt. Katastrophenmedizinische Selbstverständlichkeiten, wie Patienten-Anhängekarten, IT-Material und netzunabhängige Kommunikationsmittel, standen erst nach 6 Tagen, zum Abschluss der Evakuierungsoperation, zur Verfügung und wurden nicht einmal mehr ausgepackt. Die Ausrüstung mit Kommunikations- und Führungsmittel muss bereits in der Initialphase erhöht und verbessert werden.

Dass es auch anders gehen kann, hat die freiwillige Vorleistung der großen Fluggesellschaften und Ambulanzflieger gezeigt. Sie waren mehr als 24 Stunden früher vor Ort und haben insgesamt den Löwenanteil der Evakuierungsbewegungen bewältigt. Leider haben sich manche staatlichen Stellen zunächst schwer getan, mit den betreffenden Gesellschaften zusammenzuarbeiten und die angebotene fachliche wie sachbezogene Hilfe in ihre Bemühungen zu integrieren. Erst nachdem sich ein vertrauensvolles Miteinander vor Ort eingespielt hatte, wurden die anfänglichen Vorbehalte fallen gelassen und wichen einer fruchtbaren Zusammenarbeit. Offensichtlich war den Entscheidungsträgern kaum gegenwärtig, über welche Möglichkeiten die Airlines verfügen: Lufthansa und Condor haben neben dem normalen Linienverkehr mehr als das Doppelte der Schwerverletzten versorgt und ausgeflogen, als es die Kapazitäten der Bundeswehr erlaubten. Die erste Medevac-Maschine, welche technisch am besten für die Versorgung Schwerstverletzter geeignet war, traf am 4. Tag nach dem Ereignis ein. Das war viel zu spät. Hätte eine Erstversorgung von Patienten stattfinden müssen, wäre eine medizinische Katastrophe nach der Katastrophe die Folge gewesen.

Der zweite Kritikpunkt geht aus dem oben genannten hervor und bezieht sich auf das kommunikative Miteinander: Menschen müssen sich persönlich kennen und schätzen, um effizient miteinander arbeiten zu können. Viele Schwierigkeiten resultierten aus dem bunten Mix der Hilfsorganisationen vor Ort, der fehlenden Standardisierung katastrophenmedizinischer Prozeduren, den anfänglichen Vorbehalten staatlicher Entscheidungsträger gegen ihnen unbekannte und für sie kaum einschätzbare Hilfsangebote. Wir halten es für erforderlich, im Vorfeld einer Katastrophe ein Netzwerk miteinander kooperierender Kräfte zu schaffen, die gemeinsam eine Arbeitsebene finden und sich eng und persönlich mit nationalen wie internationalen politischen Entscheidungsträgern vernetzen. Hierzu gehört insbesondere das gemeinsame Abhalten von Übungen.

Deutschland kann es besser. Es gibt in diesem Land so große Kapazitäten an Hilfsbereitschaft, Know-how, qualifizierten Kräften und Material, dass ein hausgemachter Versorgungsengpass nicht toleriert werden muss. Es gilt, die vorhandenen Kräfte besser zu bündeln. Derartige Katastrophenlagen müssen gedanklich antizipiert und durchgeplant werden. In den politischen Krisenstäben müssen entsprechend erfahrene Experten besser vertreten sein. Für eine medizinische Großschadenslage kommt nur ein notfallmedizinisch erfahrener und praktizierender Arzt, möglichst mit Einsatzerfahrung in derartigen Lagen, in Betracht. Die relevanten Kräfte zur Bewältigung einer derartigen Schadenslage müssen identifiziert und im Vorfeld integriert werden. Bei internationalen Katastrophen diesen Ausmaßes sind dies insbesondere Bundeswehr, THW, Sanität und infrastrukturell ausreichend ausgestattete Airlines wie z.B. die Deutsche Lufthansa AG. Nur so kann das realistische Ziel erreicht werden, qualifizierte Hilfe innerhalb von 20 Stunden anstatt nach vier Tagen an einen Notfallort wie Phuket zu bringen.

Die Entscheidungsstrukturen bedürfen einer Verschlankung und Vereinfachung. Die Einsatzleitung muss vor Ort gehen. Sie muss in unmittelbarem Kontakt zu den dort arbeitenden Ärzten bleiben und einen adäquaten Verfügungsrahmen besitzen. Eine ständige Rückkopplung „nach oben“ und warten auf Bestätigungsfaxe aus Deutschland führt zu stundenlanger kontraproduktiver Verzögerung. Auf internationaler Ebene ist eine Vernetzung, das Erstellen gemeinsamer Katastrophenpläne und Abhalten gemeinsamer Übungen notwendig. So erwies es sich beispielsweise als sehr störend, dass jede Nation mit einem eigenen Team jedes Krankenhaus nach „seinen“ Patienten absuchte. Dies legte die Arbeit der thailändischen Kliniken nahezu lahm. Mittels besserer Absprachen hätte es kein Problem dargestellt, in europäischen Teams die Kliniken einmal nach europäischen Patienten zu durchsuchen. Derartige Absprachen allerdings müssen im Vorfeld einer Katastrophe getroffen werden, im Organisationschaos ist es zu spät dafür.

Jedem Anfang wohnt ein Zauber inne. Nach dieser Katastrophe gilt es, eine Menge neu aufzubauen. Nur, wer die Augen vor den bestehenden Problemen nicht verschließt, kann von sich behaupten, seiner Aufgabe gerecht zu werden. Dies aber sind wir alle den tragischen Opfern der Flutkatastrophe schuldig.

Für die Autoren:

Dr. med. Andreas Gabel, Medizinischer Dienst der Deutschen Lufthansa AG, Facharzt f. Innere Medizin, Arbeitsmedizin, Kardiologe, Notfallmedizin, Flugmedizin

\section{Notärzte in Phuket (Autoren kursiv)}

Leitende Notärzte: Vorderwülbecke, Florian, Dr. med., Arzt für Allgemeinmedizin, Deisenhofen; Gabel, Andreas, Dr. med., Medizinischer Dienst der Deutschen Lufthansa AG; Braun, Jörg, Dr. med., Medizinischer Direktor der Deutschen Rettungsflugwacht

Notärzte: BRK, Kreisverband München: Greie, Anette, Dr. med.; Laich, Anette, Dr. med.; Pischa, Ulrich, Dr. med.; Zöpf, Constanze, Dr. med.

BRK, Kreisverband Miesbach

Dambrowsky, Rolf, Dr. med.; Hertle, Iris, Dr. med.

BRK, Kreisverband Starnberg: Kirchner, Holger, Dr. med.

Krankenhaus München Schwabing: Bischoff, Wunibald, OrgLeiter; Assal, Josef, Dr. med.; Grundhuber, Hans, Dr. med.; Guggemoos, Wolfgang, Dr. med.; Hoffmann, Holger, Dr. med.; Scherer, Jörg, Dr. med.; Seemüller, Josef, Dr. med.; Thiäner, Axel, Dr. med. 